\title{
INFRAESTRUTURA DE REDES E DOS LABORATÓRIOS DE INFORMÁTICA DE ESCOLAS PÚBLICAS DE CONSELHEIRO LAFAIETE
}

\author{
Marcelo Augusto de Carvalho ${ }^{1}$ \\ ORCID: 0000-0003-2093-3601 \\ Thiago Oliveira ${ }^{2}$ \\ ORCID: 0000-0001-6675-7063
}

\begin{abstract}
Resumo: Vários projetos de inclusão digital têm sido apresentados e executados, disponibilizando computadores e acesso à Internet a escolas, porém não disponibilizam profissionais em cada escola capacitados para dar manutenção e suporte aos laboratórios. Em detrimento disso, o projeto de extensão Infraestrutura de rede e dos laboratórios de informática nas escolas públicas de Conselheiro Lafaiete (MG) tem a finalidade de garantir que todos os laboratórios estejam aptos para receber os alunos dessas escolas. Além da manutenção dos computadores, ainda há suporte semanal para alunos e professores que queiram aprender mais sobre os como utilizar o
\end{abstract}

1 Graduando do Curso de Engenharia Mecatrônica - UFSJ e bolsista do Projeto de Extensão Infraestrutura de Rede e dos Laboratórios de Informática de Escolas Públicas de Conselheiro Lafaiete, financiado por PIBEX/UFSJ.

2 Professor Adjunto da Universidade Federal de São João Del Rei (UFSJ) na área de Computação Aplicada à Engenharia do Departamento de Tecnologia em Engenharia Civil, Computação e Humanidades (DTECH) - UFSJ do Campus Alto Paraopeba. Doutor em Ciência da Computação pela Universidade Federal de Minas Gerais, concluiu o Mestrado em Ciência da Computação na instituição e obteve o título de Bacharel em Ciência da Computação também pela UFMG. 
computador, acessar a Internet e os diversos softwares e ferramentas educacionais disponíveis para uso no dia a dia escolar.

Palavras-chave: Informática. Laboratório. Manutenção. Computadores. Educação.

\title{
INFRASTRUCTURE OF NETWORKS AND COMPUTER LABORATORIES OF CONSELHEIRO LAFAIETE PUBLIC SCHOOLS
}

\begin{abstract}
Several digital inclusion projects have been presented and executed, providing computers and Internet access to schools, but they do not provide professionals in each school trained to maintain and support the laboratories. To the detriment of this, the extension project Network infrastructure and computer labs in the public schools of Conselheiro Lafaiete (MG) aims to ensure that all labs are able to receive students from these schools. In addition to computer maintenance, there is also weekly support for students and teachers who want to learn more about using the computer, accessing the Internet, and the various educational software and tools available for everyday school use.
\end{abstract}

Keywords: Informatics. Laboratory. Maintenance. Computers. Education.

\section{INFRAESTRUCTURA DE REDES Y LABORATORIOS INFORMÁTICOS DE LAS ESCUELAS PÚBLICAS DE CONSELHEIRO LAFAIETE}

Resumen: Se han presentado y ejecutado varios proyectos de inclusión digital, que proporcionan computadoras y acceso a Internet a las escuelas, pero no brindan profesionales en cada escuela capacitados para mantener y apoyar los laboratorios. En detrimento de esto, el proyecto de extensión Infraestructura de red y laboratorios de computación en las escuelas públicas de Conselheiro Lafaiete (MG) tiene como objetivo garantizar que todos los laboratorios puedan recibir estudiantes de estas escuelas. Además del mantenimiento de la computadora, también hay soporte semanal para estudiantes y maestros que desean aprender más sobre el uso de la computadora, el acceso a Internet y los diversos programas y herramientas educativas disponibles para el uso escolar diario.

Palabras claves: Informática. Laboratorio. Mantenimiento. Computadoras. Educacion.

\section{INTRODUÇÃO}

A inclusão digital consiste de uma democratização do acesso à tecnologia de informação, de modo que permita a todos usufruir do avanço tecnológico para melhorar suas condições de vida. Entre as estratégias inclusivas, estão projetos e ações que facilitam o acesso de pessoas de baixa renda às Tecnologias da Informação e Comunicação (TIC) (CULTI, 2005). Para esse fim, muitos projetos de 
inclusão têm sido apresentados e executados pelo governo, organizações não governamentais e iniciativa privada (INCLUSÃO, 2019). A maioria dos programas disponibiliza computadores e acesso à Internet a escolas, entidades assistenciais ou em local dedicado ao programa de inclusão digital. Entretanto, se esquecem de disponibilizar pessoas capacitadas para executar os programas, ou contam com voluntários para executar os projetos.

Estima-se, segundo o IBGE, que no ano de 2019 a cidade de Conselheiro Lafaiete possua 128.589 habitantes. As escolas públicas estaduais são responsáveis por boa parte da formação do ensino básico na cidade. Algumas dessas escolas dispõem de laboratórios de informática modernos sem, contudo, dispor de profissionais para dar manutenção e suporte a cada laboratório. Assim, laboratórios de informática que possuem dezenas de computadores são subutilizados, devido em parte pela carência de profissionais necessários que ofereçam apoio e suporte aos professores e alunos em cada escola, sem dispor do potencial da inclusão digital de alunos e professores.

De acordo com o mapa da inclusão digital da FGV (NERI, 2012), Conselheiro Lafaiete, aparece na $227^{\text {a }}$ posição no Brasil, com cerca 53,31\% dos domicílios que possuem microcomputadores, abaixo de sua vizinha na região do Alto Paraopeba, Ouro Branco, que tem 58.35\% de lares com microcomputadores.

A universidade tem um papel fundamental no processo de inclusão digital, pois detém o conhecimento, desde a tecnologia necessária até os métodos que devem ser utilizados para melhor aproveitamento dos recursos disponibilizados.

O programa de extensão universitária auxilia algumas escolas de Conselheiro Lafaiete (MG), realizando a inclusão digital e a manutenção dos laboratórios de informática com participação de um bolsista e um professor. Objetiva-se que todos os alunos dessas escolas, e posteriormente de todas as escolas estaduais, tenham acesso básico a computadores e Internet, além de treinamentos de introdução à Informática com material didático autoexplicativo.

Sendo assim, cada etapa do programa será apresentada detalhadamente, bem como a metodologia utilizada e os devidos resultados. O restante deste trabalho está organizado da seguinte forma. A seção 2 apresenta a metodologia. A seção 3 descreve o desenvolvimento e resultados. As conclusões do trabalho são apresentadas na seção 4 .

\section{METODOLOGIA}

Este programa é uma continuidade e expansão do projeto de inclusão digital, iniciado em 2010, onde naquele ano, um bolsista iniciou seu trabalho na Escola 
Estadual Professora Maria Augusta Noronha. Neste projeto, foi possível realizar a administração do laboratório de informática (15 máquinas), colocando-o em operação e mantendo-o disponível para alunos e professores a partir de maio de 2010. Além disso, foram realizados cursos de curta duração (12 horas) para pequenos grupos, com emissão de certificados para os alunos concluintes, que possibilitou a cerca de 40 alunos terem noções básicas de informática. Foi uma experiência muita rica e gratificante para a comunidade da escola (alunos, familiares, professores, diretores), para o aluno bolsista e para os professores da UFSJ envolvidos no projeto.

Com o passar do tempo, as técnicas utilizadas no programa foram aprimoradas e estendidas para as demais escolas públicas estaduais do município. As escolas atendidas pelo projeto de extensão atual são:

- EE Domingos Bebiano

- EE Monsenhor Horta

- EE Narciso de Queirós

- EE Prof ${ }^{\circ}$ Astor Vianna

- EE Profa Maria Augusta Noronha

O projeto atualmente é composto por um docente da UFSJ e um aluno bolsista da UFSJ. As atividades exigem $20 \mathrm{hs}$ de participação de cada bolsista.

O projeto é executado em parceria com a Superintendência Regional de Ensino (SRE), tendo como prioridade alunos que não tem acesso a computadores e à Internet, ou que possuem acesso restrito a estes recursos. O bolsista envolvido atua junto às escolas na manutenção dos computadores e dão suporte a turmas com alunos e professores. O sistema operacional utilizado nas escolas é o Linux Educacional (LINUX, 2018), versão da Secretaria Estadual de Educação de Minas Gerais (SEE-MG):

- Ambiente operacional: sistemas operacionais, sistema de arquivos, configuração básica, aplicativos básicos (antivírus, bloco de notas, calculadora, editor de imagens).

- Editores de textos: digitação, formatação, revisão ortográfica e gramatical, diagramação de tabelas, figuras e demais objetos gráficos.

- Planilha eletrônica: entradas de dados, somatório, fórmulas, gráficos e tabelas.

- Editores de apresentação: criação de slides, inserção de figuras, gráficos, vídeos e tabelas, formatação de slides e animações.

- Internet: funcionamento das redes de computadores, email, transferência de arquivos, acesso a sistemas bancários, busca de informação e segurança na Internet.

- Jogos educativos: Scratch (programação) (RESNICK, 2019), jogos que envolvem raciocínio lógico e matemático, quebra-cabeças, xadrez, tabela periódica, mapas etc.

O Programa Infraestrutura de Redes e dos Laboratórios de Informática de Escolas Públicas de Conselheiro Lafaiete auxilia também o Programa de Extensão 
Inclusão Digital nas Escolas Estaduais de Conselheiro Lafaiete, este oferece cursos básicos de informática para os alunos em algumas escolas em comum e seus bolsistas desenvolvem material próprio, como uma apostila foi desenvolvida e disponibilizada via plataforma Moodle (SABBATINI, 2007), um ambiente virtual de aprendizagem de código livre, aberto e gratuito que oferece recursos avançados para a prática de ensino a distância $(\mathrm{EaD})$. Desta forma, os bolsistas podem se dedicar aos alunos que tem mais dificuldade enquanto outros alunos, que por ventura já conhecem o tópico tratado ou tem facilidade com informática, podem avançar sozinhos em seu estudo. O formato multimídia da apostila funciona como um motivador para os alunos se comparado com a versão estática que normalmente seria usada nestes cursos.

Por fim, uma pesquisa de satisfação é feita com alunos e professores de cada escola, a fim de serem obtidas informações e opiniões a respeito do projeto e a situação dos laboratórios de informática.

\section{DESENVOLVIMENTO E RESULTADOS}

Em relação à manutenção dos laboratórios de informática e sua infraestrutura, podemos ver o desempenho do programa no gráfico da Figura 1.

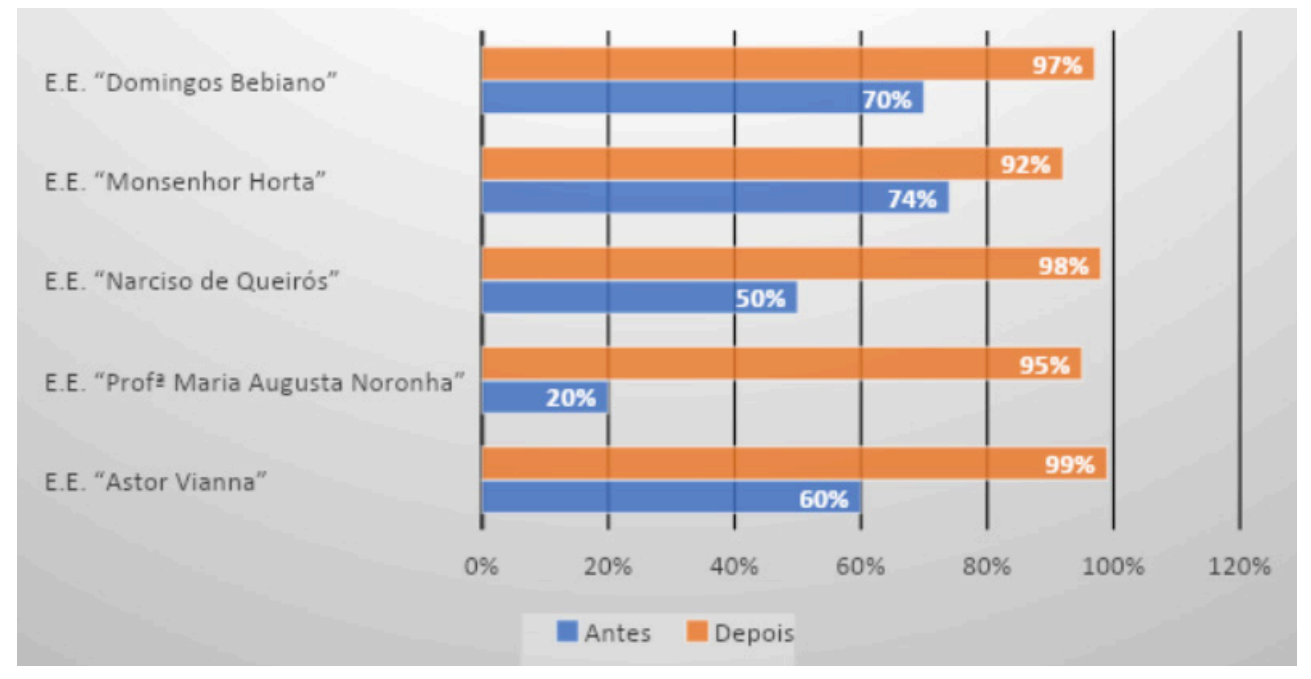

FIGURA 1. TAXA DE OPERACIONALIZAÇÃO DOS LABORATÓRIOS E MELHORIAS DO PROGRAMA.

O número de máquinas por laboratório das escolas variou de acordo com o planejamento de cada uma em manter máquinas mais antigas de outras licitações. Quando as escolas decidem manter máquinas de licitações antigas, aumenta o número de máquinas. Porém em muitos casos são máquinas antigas que não 
tem suporte para o Sistema Operacional atual ou não se acham peças para reposição, impossibilitando colocar o laboratório 100\% funcional.

A disponibilização do laboratório para os alunos 4 horas por semana, para pesquisas e aulas diferenciadas, transforma o computador antes inútil num poderoso recurso de suporte a aprendizagem com inúmeras possibilidades pedagógicas.

O treinamento e a emissão de declarações de participação do projeto de extensão em Inclusão Digital também ajudaram nos resultados positivos. Houve tanto a conscientização sobre o uso dos instrumentos computacionais no dia-a-dia, quanto o ingresso desses alunos no mercado de trabalho, que exige cada vez mais o domínio de um computador.

A integração da universidade com a comunidade da escola facilitou a comunicação dos mesmos, trazendo melhorias para ambas as partes.

- E.E. "Prof. Astor Vianna”: A melhoria do laboratório foi de $20 \%$ para cerca de $99 \%$ após o recebimento do programa. A escola possui cursos técnicos, tanto na área de T.I. quanto em outras áreas, o que requer um uso frequente do laboratório de informática e seus recursos. Então, o bom funcionamento do laboratório é algo decisivo para que o conteúdo e práticas sejam bem realizados pelos professores e alunos. Os alunos do ensino fundamental e ensino médio, assim como o ensino integral, também faziam uso do laboratório durante a manhã de forma razoável. Além disso alguns professores e funcionários faziam uso dos computadores em seus afazeres diários.

- E.E. "Prof.a Maria Augusta Noronha": Foi a escola com resultado mais expressivo, saindo de $20 \%$ para $95 \%$ dos computadores funcionando. Antes do início do programa na escola, o laboratório encontrava-se sucateado. Os computadores e mesas todos espalhados e jogados pela sala. Praticamente nada funcionava e foi necessário um tempo maior de trabalho para que voltasse a ser utilizado pela escola. A manutenção foi um sucesso e passou a ser utilizados novamente pelos alunos e professores, mesmo que de forma não tão frequente, mas que certamente fez a diferença, pois muitos deles não tinham acesso ao computador na residência.

- E.E. "Narciso de Queirós": O laboratório encontrava-se 50\% funcional e foi para 97\%. Os professores não utilizavam o laboratório de informática devido à falta de internet, inicialmente. Após o recebimento do programa, o problema da internet foi resolvido e alguns professores passaram a levar os alunos para o laboratório, principalmente os alunos de ensino integral, para fazerem pesquisas e outros trabalhos.

- E.E. "Monsenhor Horta": O laboratório passou de 74\% para 92\% operacional. Entre as escolas atendidas, é a que possui o maior número de computadores funcionais. O laboratório é mais frequentemente utilizado pelos alunos e 
professores, principalmente durante o intervalo. A escola possui um projeto em que os alunos escrevem um ebook e muitas das vezes fazem isso no laboratório de informática durante o intervalo. Logo, a escola pode contar com o apoio do bolsista também nesta atividade.

- E.E. "Domingos Bebiado": O laboratório da escola saiu de 70\% para 97\% funcional. O laboratório não possui muitos computadores, mas atende bem a escola, uma vez que não é utilizado frequentemente. Alguns professores levam os alunos para mostrar de forma prática o conteúdo da disciplina e pesquisas adicionais.

Dificuldades encontradas: a principal dificuldade encontrada foi a falta de vontade e resistência dos professores a utilizarem o laboratório de informática, seja por comodismo ou mesmo falta de conhecimento básico de informática. Já na parte de infraestrutura de redes, muitas vezes as empresas de telecomunicação responsável pela rede das escolas e até mesmo os técnicos da superintendência não oferecem serviços de qualidade, nesses casos coube ao bolsista mostrar um ponto de vista diferente fazendo um contato direto na tentativa de solucionar eventuais problemas da rede das escolas. Além disso, a falta de recursos fez com que não fosse possível tornar o laboratório $100 \%$ funcional, pois há a falta de certos materiais e periféricos.

Em relação à pesquisa de satisfação, os resultados são mostrados a seguir nas figuras 2 a 5 na escola "Astor Vianna" e assim sucessivamente. Laboratório foi avaliado como subutilizado e $73 \%$ o consideram bom ou ótimo.

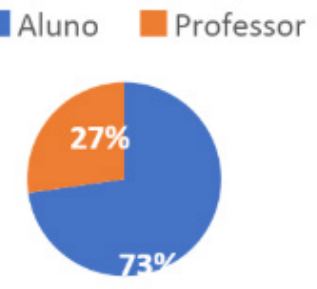

Figura 2. Quantidade de Alunos e Professores.

\section{Alunos}

8

\section{Professores}

FIGURA 3. ESTADO do LABORATÓRIO. 


\begin{tabular}{|c|c|c|c|c|c|}
\hline Ótımo & Bom & RazoÁvel & RuIM & Péssimo & Nunca utilizei \\
\hline 4 & 4 & 1 & 0 & 0 & 2 \\
\hline
\end{tabular}

Uso do laboratório

— Frequentemente utilizado Subutilizado

FIGURA 4. USO DO LABORATÓRIO

\begin{tabular}{|c|c|}
\hline FREQUENTEMENTE UTILIZADO & SUBUTILIZADO \\
\hline 4 & 7 \\
\hline
\end{tabular}

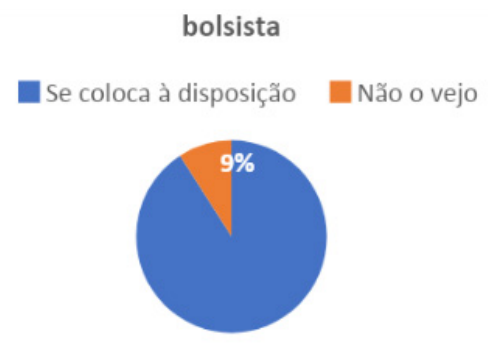

Figura 5. ATIVIDAdE do Bolsista.

SE COLOCA À DISPOSIÇÃo

NÃO O VEJo

10

1

Em relação à pesquisa de satisfação, os resultados são mostrados a seguir nas figuras 6 a 9 na escola "Maria Augusta Noronha". Laboratório subutilizado e 60\% avaliam bem.

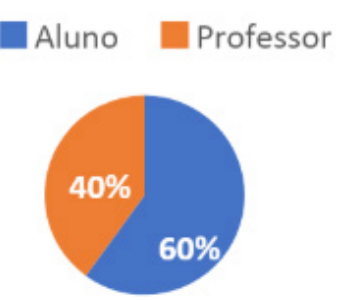

Figura 6. QuANtidade de Alunos e Professores.

\section{Aluno}

9
Professor

6 


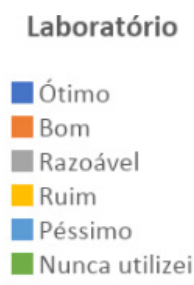

FiguRA 7. ESTADO dO LABORATÓRIO.

\begin{tabular}{|c|c|c|c|c|c|}
\hline Ótımo & Bom & RazoÁvel & Ruim & Péssımo & NunCA Utilizei \\
\hline 3 & 6 & 2 & 0 & 0 & 4 \\
\hline
\end{tabular}

Uso do laboratório

arequentemente utilizado Subutilizado

FIGURA 8. USO DO LABORATÓRIO.

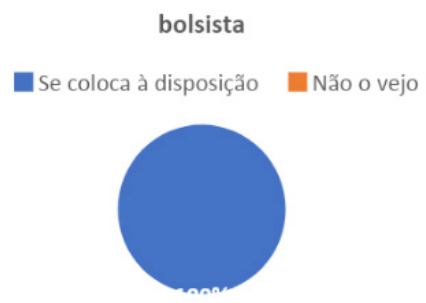

Figura 9. ATIVIDADE DO Bolsista.

SE COLOCA À DISPOSIÇÃo

NÃO O VEJO

Em relação à pesquisa de satisfação, os resultados são mostrados a seguir nas figuras 10 a 13 na escola "Narciso de Queirós". Laboratório subutilizado e 54\% avaliam bem. 
Figura 10. Quantidade de Alunos e Professores.

\section{Aluno}

\section{PROFESSOR}

FIGURA 11. ESTADO DO LABORATÓRIO.

\begin{tabular}{|c|c|c|c|c|c|}
\hline Ótımo & Bom & RazoÁvel & RuIM & Péssımo & Nunca Utilizei \\
\hline 2 & 5 & 2 & 0 & 0 & 4 \\
\hline
\end{tabular}

Uso do laboratório

Grequentemente utilizado Subutilizado

FIGURA 12. USO DO LABORATÓRIO.

FREQUENTEMENTE UTILIZADO
Laboratório

Q́timo

Bom

Razoável

Ruim

Péssimo

Nunca utilizei

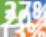

\begin{tabular}{|c|c|}
\hline FREQUENTEMENTE UTILIZADO & SUBUTILIZADO \\
\hline 1 & 12 \\
\hline
\end{tabular}

bolsista

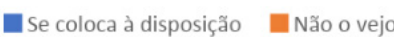

Figura 13. AtividAde do Bolsista.

SE COLOCA À DISPOSIÇÃO

NÃo o VEJo 
Em relação à pesquisa de satisfação, os resultados são mostrados a seguir nas figuras 14 a 17 na escola E.E. "Monsenhor Horta". Laboratório subutilizado, 59\% o consideram ótimo e 37\% consideram bom.

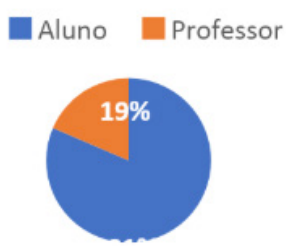

Figura 14. Quantidade de Alunos e Professores.

\begin{tabular}{|c|c|}
\hline ALUNO & Professor \\
\hline 22 & 5 \\
\hline
\end{tabular}

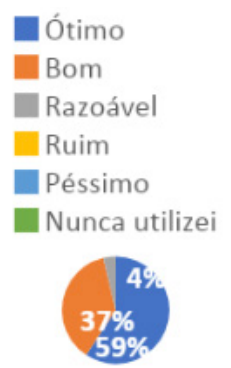

Figura 15. ESTADO DO LABORATÓRIO.

\begin{tabular}{|c|c|c|c|c|c|}
\hline Ótıмo & Bom & RAzó́vel & RuıM & PÉssımo & NUNCA UtILIzEI \\
\hline 16 & 10 & 1 & 0 & 0 & 0 \\
\hline
\end{tabular}

Uso do laboratório

—Frequentemente utilizado $\quad$ Subutilizado

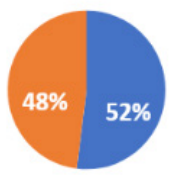

FIGURA 16. USO DO LABORATÓRIO.

FREQUENTEMENTE UTILIZADO

12
SUbUtiLIzAdo

12

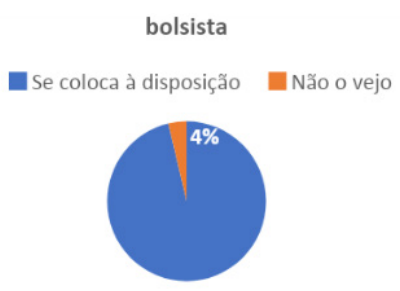

Figura 17. Atividade do Bolsista. 
Em relação à pesquisa de satisfação, os resultados são mostrados a seguir nas figuras 18 a 21 na escola "Domingos Bebiano". Laboratório subutilizado e 67\% consideram bom.

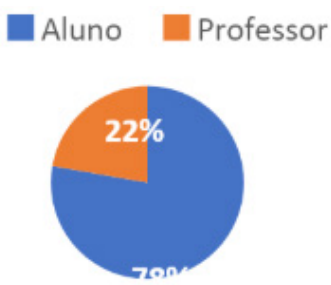

Figura 18. QuANTIDADE DE Alunos e Professores.

Aluno

7

\section{PROFESSOR}

FIGURA 19. ESTADO DO LABORATÓRIO.

\begin{tabular}{|c|c|c|c|c|c|}
\hline ÓtıMo & Bom & Razoável & RuIM & Péssimo & Nunca Utilizel \\
\hline 1 & 6 & 0 & 0 & 0 & 2 \\
\hline
\end{tabular}

Frequentemente utilizado Subutilizado

FIGURA 20. USO NO LABORATÓRIO.

FREQUENTEMENTE UTILIZADO

\section{Subutilizado}

8 


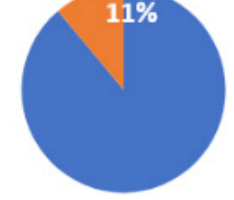

FIGURA 21. ATIVIDADE DO Bolsista.

SE COLOCA À DISPOSIÇÃO
NÃO O VEJO

TOTAL DE RESPOSTAS

90

O programa foi publicado em diversos meios de comunicação de Conselheiro Lafaiete, enfatizando a parceria entre a UFSJ e a Secretaria de Educação - que beneficiou algumas escolas públicas da cidade, sem custos. Este projeto foi apresentado no $7^{\circ}$ Congresso Brasileiro de Extensão Universitária - CBEU (Anais, 2016) em Ouro Preto - MG, além de ter apresentado os resultados iniciais no ano de 2011 nesse congresso sediado na cidade de Porto Alegre - RS, resultando em uma rica troca de experiências, apresentação de propostas e resultados e oportunidade de refletir criticamente sobre a extensão universitária no Brasil.

Ao final do ano letivo, alguns alunos e professores foram submetidos de forma voluntária a um questionário sobre o laboratório de informática da escola. Após a análise das respostas, apresentadas nos gráficos e descrições na seção correlata aos resultados, é possível ter-se a visão do laboratório de informática pela perspectiva do aluno e do professor, evidenciando que o programa se faz presente na escola e laboratório atende bem à demanda dos alunos e professores, porém o desejo de ser mais frequentemente utilizado ainda se faz presente, bem como seus recursos e ferramentas.

Do ponto de vista do bolsista, houve a aplicação e complementação de sua formação como estudante de graduação junto à comunidade acadêmica, auxiliando também no desenvolvimento manutenção da infraestrutura básica de ensino das escolas de sua cidade, além da satisfação de poder retribuir através do serviço às escolas nas quais fez o ensino fundamental e médio.

\section{CONCLUSÃO}

Após os resultados obtidos por meio da comparação entre a situação inicial analisada nos laboratórios de informática, evidenciados nos gráficos da seção correlata 
aos resultados, observa-se que foi possível valorizar os laboratórios de informáticas das escolas abrangidas pelo programa. Na E.E."Maria Augusta Noronha", por exemplo, a taxa de operacionalização do laboratório cresceu de $20 \%$, quando o programa iniciou na escola, para 95\% após a realização de todas as etapas do programa. Assim também as outras escolas tiveram resultados semelhantes, onde o laboratório de informática passou a ser funcional e consequentemente mais utilizado pelos professores e alunos. Logo a disponibilidade dos laboratórios passou a ser garantida, com isso alguns professores passaram a levar alunos com mais frequência para o laboratório. Além disso, foi possível despertar o interesse dos alunos pela tecnologia e pelo conhecimento sobre o mundo digital também para professores.

Com o programa, foi possível entender um pouco sobre a realidade da comunidade da escola, perceber seus valores sociais e apresentar um pouco sobre os cursos existentes no Campus Alto Paraopeba (UFSJ, 2019) aos alunos que prestarão o Enem. Ao mesmo tempo, foi possível ter a satisfação de obter resultados para os alunos e para a Escola, aproximando a Universidade da comunidade onde está inserida.

Nas escolas onde não houve a possibilidade de ser ofertado o treinamento em conjunto com a manutenção dos computadores, foi observado que, mesmo os computadores estando em funcionamento, não houve grande acesso ao laboratório por parte dos professores e alunos em comparação onde havia também o treinamento. Além disso, através do levantamento feito de todos os computadores de cada laboratório, foi possível um melhor acompanhamento por parte dos funcionários da escola e da SRE, o que fez com que as medidas cabíveis a estes fossem tomadas de forma mais rápida e facilitada, como a instalação da nova versão do Linux Educacional e acesso à Internet.

\section{REFERÊNCIAS BIBLIOGRÁFICAS}

Anais do $7^{\circ}$ Congresso Brasileiro de Extensão Universitária CBEU. Ouro Preto, 2016. Disponível em: http://www.cbeu.eventsystem.com.br/anais. Acesso em 07 dez. 2019.

CUlTI, M. N.; DELGADO, M. V. M. INCLUSÃO DIGITAL: A Necessidade de Ações Coordenadas. Observatório da Sociedade da Informática, UNESCO, v. 1, p. 4-4, 2005.

INCLUSÃO. Portal de Programa Nacional de Inclusão Digital. Disponível em: http://www.inclusaodigital.gov.br. Acesso em 03 fev. 2019. 
LINUX. E-Book Aplicativos Educacionais Linux See-MG. Belo Horizonte, 2018. Disponível em: https://portaldtae.educacao.mg.gov.br/index.php/materiais/central/download/9-apostilas-guias-e-manuais/32-ebook-linux-seemg. Acesso em 07 dez. 2019.

NERI, Marcelo Cortes. Mapa da Inclusão Digital. Rio de Janeiro, FGV, CPS, 2012. Disponível em: http://www.cps.fgv.br/cps/telefonica/. Acesso em 03 dez. 2019.

RESNICK, Mitch. Welcome to the ScratchEd Online Community Archive. Disponível em: http://scratched.gse.harvard.edu/. Acesso em 07 dez. 2019.

SABBATINI, Renato Marcos Endrizzi. Ambiente de Ensino e Aprendizagem via Internet - A Plataforma Moodle. 2007. Disponível em: http://www.ead. edumed.org.br/file.php/1/PlataformaMoodle.pdf. Acesso em 03 dez. 2019.

UFSJ. Campus Alto Paraopeba. Disponível em: https://www.ufsj.edu.br/cap/. Acesso em 03 dez. 2019. 